van der Pols J, Spencer L, Baade P. Colorectal cancer incidence in Australia before and after mandatory fortification of bread flour with folic acid. Public Health Nutrition [Accepted 3rd February 2021]

\title{
1 Colorectal cancer incidence in Australia before and after mandatory fortification of \\ 2 bread flour with folic acid
}

\section{Abstract}

4 Objective: Mandatory fortification of bread flour with folic acid has helped to reduce

5 incidence of neural tube defects in several countries. However, it has been suggested that

6 folic acid may have potential adenoma-promoting effects, and reports from some countries

7 have suggested that mandatory folic acid food fortification programmes have increased the

8 incidence of colorectal cancer. The objective of this study was to evaluate colorectal cancer

$9 \quad$ incidence patterns before and after introduction of mandatory folic acid fortification of bread

10 flour in Australia in 2009.

Design: Data from the Australian Cancer Database was used to plot age-standardised incidence of colorectal cancer. We calculated age-adjusted rate ratios with $95 \%$ confidence intervals.

Setting: Australia

Participants: We used population-level aggregate data obtained from cancer registries.

Results: Age-standardised colorectal cancer incidence generally decreased between 19992016. Although there was a slight increase in rates in 2010 compared to 2009 (62.8 vs. 61.6 cases per 100,000, age-adjusted rate ratio 1.02 (95\% CI 0.99-1.04), joinpoint regression indicated decreases of $-0.4 \%(95 \% \mathrm{CI}-0.7-0.0)$ per year from 1999 to 2010 and $-2.2 \%$ (95\%CI -3.1--1.3) per year from 2010 to 2016.

Conclusions: While causation cannot be assessed from these population-level data, our observations indicate that there is no evidence that introduction of mandatory folic acid fortification of bread flour has influenced colorectal cancer incidence in Australia. 
Mandatory fortification of bread flour with folic acid is currently being considered by governments of the $\mathrm{UK}^{(1)}$ and New Zealand ${ }^{(2)}$. Data from other countries have shown that such fortification programmes can help achieve significant reductions in the incidence of neural tube defects, in particular in high-risk population groups ${ }^{(3)}$. Despite these confirmed public health benefits, some countries have hesitated to implement mandatory folic acid fortification for a number of reasons, including suggestions of a possible increased incidence of colorectal cancer ${ }^{(4,5)}$, due to potential adenoma-promoting effects of folic acid fortification $^{(6)}$. The timing of increases in colorectal cancer incidence rates in USA and Canada have been provided as evidence supporting the role of folic acid in increasing colorectal cancer risk ${ }^{(5)}$. A recent USA expert workshop identified priorities for continued research of the potential adverse health effects of excess folate or folic acid intake ${ }^{(7)}$.

Bread flour in Australia has been fortified with folic acid since September 2009. Monitoring of this mandatory fortification has shown good compliance ${ }^{(8)}$. While ecological data analyses have obvious limitations with assessing causality, to date Australian trends in colorectal cancer incidence have not been considered in relation to the introduction of mandatory bread flour fortification. We therefore used population-level data to evaluate colorectal cancer incidence patterns before and after introduction of mandatory folic acid fortification of bread flour in Australia.

\section{Methods}

We used data from the Australian Cancer Database, published by the Australian Institute of Health and Welfare ${ }^{(9)}$. These data include all new cases of primary invasive colorectal cancers (ICD codes C18-C20) diagnosed between 1999-2016 (the most recent data available). We plotted age-standardised incidence of colorectal cancer (cases per 100,000, standardised 
to the 2001 Australian population) and calculated age-adjusted rate ratios with $95 \%$ confidence intervals $(95 \% \mathrm{CI})$ using the method by Fay and Feuer ${ }^{(10)}$. Age-group specific incidence rate ratios with $95 \% \mathrm{CI}$ were calculated using a negative binomial regression model. Joinpoint Trend Analysis Software version 4.8.0.1 was used to analyse trends ${ }^{(11)}$.

\section{Results}

Age-standardised colorectal cancer incidence generally decreased between 1999-2016 (Fig. 1a), although there was a slight increase in rates in 2010 compared to 2009 (62.8 vs. 61.6 cases per 100,000, age-adjusted rate ratio 1.02 (95\%CI 0.99-1.04) (Fig. 1b). Age-specific patterns (Fig.2) indicated that the slight increase in 2010 vs. 2009 was mainly due to a higher incidence in people aged 55-59 yr (99.0 vs. 88.1 per 100,000; incidence rate ratio 1.12 (95\%CI 1.04-1.22)) and 65-69 yr (229.9 vs. 210.9 per 100,000; incidence rate ratio 1.09 (95\%CI 1.02-1.16)), while other age groups changed very little (data not shown). Trend analysis indicated a joinpoint in 2010, with the significant decrease in incidence observed between 1999 and 2010 (Annual Percent Change: -0.4\% (95\%CI -0.7-0.0)) becoming more pronounced between 2010 and 2016 (Annual Percent Change : -2.2\% (95\%CI -3.1--1.3)).

\section{Discussion}

While causation cannot be assessed from these population-level data, our observations indicate that there is no evidence that introduction of mandatory folic acid fortification of bread flour in 2009 has influenced colorectal cancer incidence in Australia. The two age subgroups in which colorectal cancer incidence was slightly higher in the year following introduction of mandatory fortification (55-59 and 65-69 years), were affected by suspension of the national bowel cancer screening program in May 2009, due to faulty faecal occult 
73 blood tests ${ }^{(12)}$. At that time, people aged 50, 55 and 65 were eligible to participate in the bowel cancer screening program. The screening program was resumed in November 2009, and affected person were re-invited to be screened. Combined this may have had the effect of artificially reducing 2009 incidence and increasing 2010 incidence. This is consistent with other countries where changes in bowel cancer screening participation ${ }^{(13)}$ had been noted in response to concerns about possible negative effects of folic acid fortification programs on colorectal cancer incidence.

Thus, the small fluctuations in colorectal cancer incidence around the year of introduction of mandatory folic acid fortification, coincide with known variations in screening participation.

These variations may also be due to normal, random year-to-year variation of colorectal cancer incidence observed in populations. For most cancer risk factors, there is a lag period between exposure to the risk factor and the subsequent diagnosis of cancer. Indeed, estimates of the latency period between exposure to risk factors and colorectal cancer diagnosis vary widely from 4 to 20 years ${ }^{(14,15)}$ Given this, it would be surprising if a fortification-induced increase in the population incidence of colorectal cancer incidence were observed in the year after its introduction. Our trend analysis indicated an acceleration in the rate of decline in colorectal cancer incidence in the seven years following implementation of mandatory folic acid fortification. The continued decrease in colorectal cancer incidence rates after 2010 provides further evidence against a negative impact of folic acid fortification.

These findings appear to contrast with observations in $\operatorname{Iran}^{(16)}$, though direct comparisons with other countries is difficult for example due to differences in fortification regimes and different colorectal cancer surveillance practices.

In conclusion, while definitive evidence likely requires the establishment of large-scale cohort studies or randomised controlled trials, our population-level findings are consistent with the position by Cancer Council Australia and the Cancer Society of New Zealand that 
98 folic acid fortification of bread flour for the prevention of neural tube defects does not

99 adversely affect colorectal cancer incidence ${ }^{(17)}$. Increasing the scope of quantitative data at 100 the individual and population level about the impact of folic acid on colorectal cancer

101 internationally should remain a priority, to ensure that decisions made by health agencies are 102 made on the best available evidence.

103 


\section{REFERENCES}

1. Department of Health and Social Care UK (2019) Proposal to add folic acid to flour: consultation document. https://www.gov.uk/government/consultations/adding-folic-acid-to$\underline{\text { flour/proposal-to-add-folic-acid-to-flour-consultation-document }}$

2. New Zealand Food Safety - Ministry for Primary Industries (2019) Folic acid fortification: Technical supporting document. Technical Paper No: 2019/04.

3. Hilder L (2016) Neural Tube Defects in Australia, 2007-2011: Before and after implementation of the mandatory folic acid fortification standard. Publication Number 11488. National Perinatal Epidemiology and Statistics Unit, University of New South Wales, Australia.

4. Hirsch S, Sanchez H, Albala C et al. (2009) Colon cancer in Chile before and after the start of the flour fortification program with folic acid. Eur J Gastroenterol Hepatol 21, 436-439.

5. Mason JB, Dickstein A, Jacques PF et al. (2007) A Temporal Association between Folic Acid Fortification and an Increase in Colorectal Cancer Rates May Be Illuminating Important Biological Principles: A Hypothesis. Cancer Epidemiol Biomarkers Prev 16, 1325-1329.

6. Cole BF, Baron JA, Sandler RS et al. (2007) Folic Acid for the Prevention of Colorectal Adenomas: A Randomized Clinical Trial. JAMA 297, 2351-2359.

7. Maruvada P, Stover PJ, Mason JB et al. (2020) Knowledge gaps in understanding the metabolic and clinical effects of excess folates/folic acid: a summary, and perspectives, from an NIH workshop. The American journal of clinical nutrition 112, 1390-1403.

8. Food Standards Australia New Zealand (2016) Monitoring of folic acid fortification https://www.foodstandards.gov.au/science/monitoringnutrients/monitoringfort/Pages/default.a $\underline{\text { spx }}($ accessed 17 December 2020)

9. Australian Institute of Health and Welfare (2020) Cancer data in Australia. https://www.aihw.gov.au/reports/cancer/cancer-data-in-australia/data

10. Fay MP, Feuer EJ (1997) Confidence intervals for directly standardized rates: a method based on the gamma distribution. Stat Med 16, 791-801. 
11. National Cancer Institute (2020) Joinpoint Trend Analysis Software.

12. Department of Health and Ageing (2012) Review of the National Bowel Cancer Screening Program (Phase 2) - Final report part one. KPMG.

13. Keum N, Giovannucci EL (2014) Folic acid fortification and colorectal cancer risk. Am J Prev Med 46, S65-72.

14. Lee JE, Willett WC, Fuchs CS et al. (2011) Folate intake and risk of colorectal cancer and adenoma: Modification by time. American Journal of Clinical Nutrition 93, 817-825.

15. Shield KD, Freisling H, Boutron-Ruault MC et al. (2018) New cancer cases attributable to diet among adults aged 30-84 years in France in 2015. British Journal of Nutrition 120, 11711180.

16. Moazzen S, Dastgiri S, Dolatkhah R et al. (2020) Staple Food Fortification with Folic Acid and Iron and Gastrointestinal Cancers: Critical Appraisal of Long-Term National Fortification. Nutr Cancer, 1-5.

17. Cancer Council Australia and Cancer Society of New Zealand (2014) Position statement Folate and reducing cancer risk. https://wiki.cancer.org.au/policy/Position_statement_Folate and reducing_cancer_risk 
Figure 1 Age-standardised incidence rates (95\% confidence intervals) for colorectal cancer in Australia. (a) Colorectal cancer incidence per 100,000 in 1999-2016. (b) A non-zero intercept detail of Figure1 (a).

Figure 2 Colorectal cancer incidence by age-group in Australia, 1999-2016. 
Figure 1 Age-standardised incidence rates (95\% confidence intervals) for colorectal cancer in Australia. (a) Colorectal cancer incidence per 100,000 in 1999-2016. (b) A non-zero intercept detail of Figure1 (a).
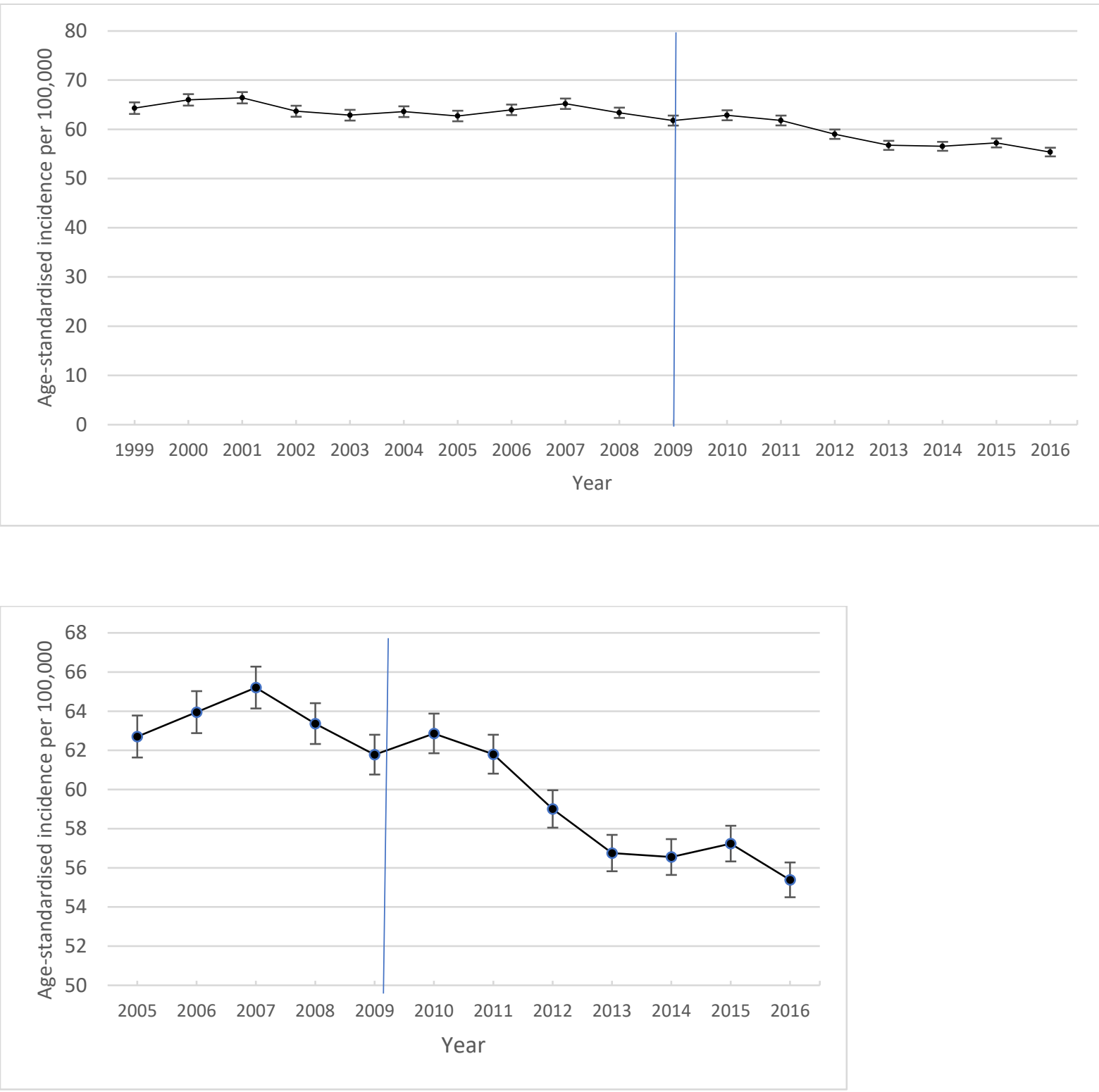
Figure 2 Colorectal cancer incidence by age-group in Australia, 1999-2016.

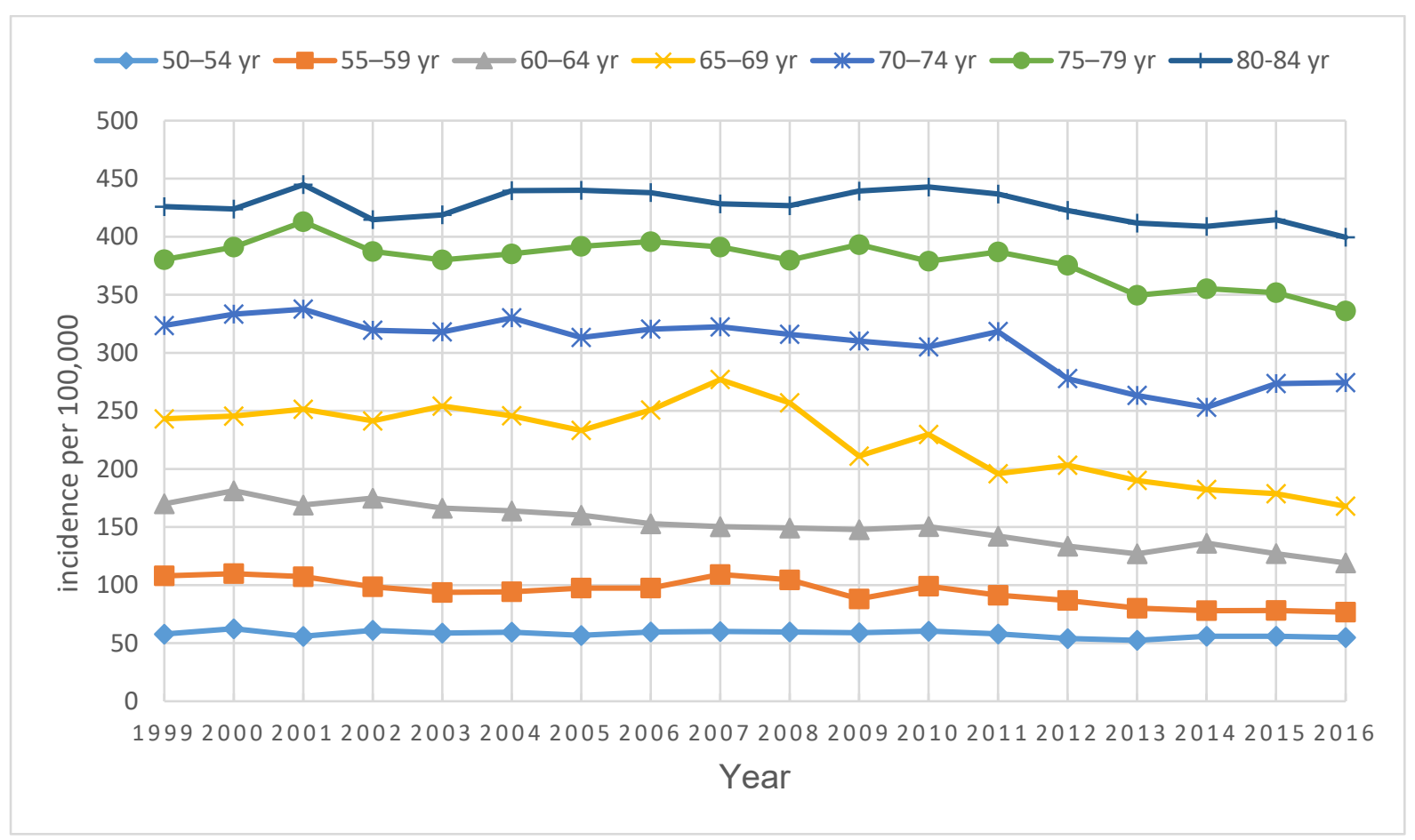

